\title{
A need for intervention: Childhood adversities are a significant determinant of health-harming behavior and poor self-efficacy in patients with alcohol dependence. An observational, cross-sectional study on the population of Central Poland
}

\author{
Dominika Berent ${ }^{1, A-D, F}$, Michał Podgórski ${ }^{2, C, F}$, Andrzej Kokoszka ${ }^{1, E, F}$ \\ ${ }^{1}$ Department of Psychiatry II, Medical University of Warsaw, Poland \\ ${ }^{2}$ Department of Diagnostic Imaging, Polish Mother's Memorial Hospital Research Institute, Łódź, Poland \\ A - research concept and design; $\mathrm{B}$ - collection and/or assembly of data; $\mathrm{C}$ - data analysis and interpretation; \\ $D$ - writing the article; $E$ - critical revision of the article; $F$ - final approval of the article
}

\section{Address for correspondence}

Dominika Berent

E-mail: dominikaberent@poczta.fm

\section{Funding sources}

None declared

Conflict of interest

None declared

\section{Received on November 4, 2016}

Reviewed on April 11, 2017

Accepted on April 27, 2017

DOI

10.17219/acem/70792

Copyright

Copyright by Author(s)

This is an article distributed under the terms of the

Creative Commons Attribution Non-Commercial License

(http://creativecommons.org/licenses/by-nc-nd/4.0/)

\begin{abstract}
Background. Adverse childhood experiences (ACEs) are known to be associated with a lasting effect on physical and psychological well-being in adulthood. Patients with alcohol dependence (AD) are a particular clinical subgroup who report a higher number of ACE categories than the general population and who develop several health-harming behaviors and poor social skills.
\end{abstract}

Objectives. To our knowledge, this is the first study on patients with AD that aimed to assess whether ACEs correlate with health habits and general self-efficacy in adulthood.

Material and methods. The study comprised 196 patients with $A D(F=50)$ with a mean age of 43.8 years. The following research tools were used: the Health Behavior Inventory (HBI), the Generalized Self-Efficacy Scale (GSES) and the ACE Study score, expanded with 3 more questions about exposure to sudden stress and violence outside the family. Additionally, the patients' sociodemographic and clinical characteristics were assessed and included in the multiple stepwise regression analysis for variation in health habits and general self-efficacy.

Results. The mean scores of the patients were $64.4 \pm 16.6$ points on the HBl and $28.4 \pm 6.2$ points on the GSES. The study revealed a mean number of $3.3 \pm 2.7$ ACEs. The multiple regression analysis showed that the ACEs were significantly and inversely associated with self-efficacy assessed by the GSES and with health habits evaluated by the HBI $(\beta=-0.377 ; p=0.026$ and $\beta=-1.210 ; p=0.007$, respectively). The ACEs accounted for $3.2 \%$ of the GSES model variability and $3.9 \%$ of the HBI variability.

Conclusions. Adverse childhood experiences might promote the development of health-harming behaviors and inferior general self-efficacy in adult patients with AD. The study suggests the need for primary and secondary preventive strategies targeted at ACEs and at general self-efficacy impaired by childhood adversities for further better well-being. However, although the influence of the ACEs was significant, there are many other factors that were not included in the analysis, which explain the remaining variability of health behaviors and general self-efficacy.

Key words: health, alcoholism, child abuse, adult survivors, self-efficacy 


\section{Introduction}

Emotional and interpersonal impairments continue to be studied in the area of alcohol dependence (AD) because of their role in treatment outcomes and general wellbeing in patients with AD. The literature states that social disabilities in patients with AD may be either primary or secondary to alcohol misuse and may be produced by both social and biological factors. ${ }^{1-3}$ Patients with AD were found to present a loss of behavioral flexibility, significant impairment in facial emotion recognition and a generally impaired ability to recognize one's own and others' mental states. ${ }^{1,2}$ Patients with AD and impairment in facial emotion recognition were found to consider alcohol misuse as a tool for improving social abilities. ${ }^{3}$ However, alcohol misuse, especially in adolescence, was found in animal studies to result in lifelong neurobiological changes and a permanent loss of hippocampal neurogenesis with the further phenotype result of impairment in behavioral flexibility. ${ }^{1}$

General self-efficacy, which is an important measure of social ability, is referred to as global confidence in one's coping ability in demanding or new situations, which require a variety of stressful experiences to be dealt with and which characterize one's social skills. ${ }^{4}$ As was noted by Bandura, patients who have strong self-efficacy are likely to mobilize the effort needed to successfully resist situations which put them at a high risk for using alcohol. ${ }^{5}$ Skill-oriented self-efficacy, i.e., the ability to not drink in high-risk situations, is associated with the effective use of coping strategies and lower relapse rates. ${ }^{6,7}$ General self-efficacy may be influenced by adverse childhood experiences (ACEs), understood as physical, emotional and sexual abuse, loss of attachment figures, and sudden potentially traumatic events, i.e., witnessing someone's death. Bandura et al. pointed out that there is a bimodal association between life adversities and self-efficacy. ${ }^{8}$ Strong selfefficacy supports recovery from traumatic experiences, but self-efficacy may be impaired by previously experienced life adversities. ${ }^{8}$ Reports in the literature indicate that ACEs may trigger adverse adaptation following traumatic experiences, and impair self-efficacy. ${ }^{9-11}$

Individuals with $\mathrm{AD}$ were found to report a higher number of ACEs than the general population. ${ }^{12,13}$ As compared with people with an ACE Study score of 0, those with an ACE Study score of 4 or more were 7 times more likely to suffer from AD. ${ }^{12}$ It was postulated that this may be due to having been raised in a dysfunctional household; however, the correlation is obviously not absolute. ${ }^{14,15} \mathrm{Nev}$ ertheless, a national study in Great Britain on ACEs found that individuals with $\geq 4$ ACEs (vs 0 ACEs) were at an approx. 3 times higher risk of developing any disease before 70 years of age, e.g., cancer, diabetes or stroke. ${ }^{16}$ This may be due to the fact that patients with $\mathrm{AD}$, apart from drinking alcohol, develop multiple health-harming behaviors, e.g., poor diet, low physical activity or cigarette smoking. ${ }^{17}$ The results of studies on the association between
ACEs and general health in adulthood based on the ACE Study score have been widely published. ${ }^{12,13,18,19}$ However, exposure to sudden stress and violence outside the family is also considered to be an important predictor of health in adulthood. ${ }^{20}$ Thus, we posed 3 more questions to our participants concerning them potentially witnessing a family member's attempted suicide, a family member's death from any cause or a stranger's death from any cause (e.g., a traffic accident).

Both health habits and general self-efficacy are contributors to individual mental and physical well-being; therefore, searching for the factors that may trigger them is still necessary in order to propose possible preventive strategies for better overall health. ${ }^{8,21}$ To our knowledge, this is the first study to assess the possible influence of childhood adversities on health habits and general self-efficacy in patients with AD. Here, we hypothesize that ACEs may promote the adoption of health-harming behaviors and may impair general self-efficacy in patients with AD.

\section{Material and methods}

\section{Study participants}

This is an observational, cross-sectional study that was performed in Poland between 2013 and 2015. A total of 209 consecutive patients with AD who were admitted to psychiatric wards for a course of AD psychotherapy or treatment of alcohol withdrawal syndrome and who gave informed consent were involved in the study. Of these patients, 13 did not undergo further analysis because of incomplete data or the withdrawal of their consent during the study. The study analyzed 196 patients with $\mathrm{AD}(\mathrm{F}=50)$ aged $43.8 \pm 10.7$ years (mean \pm SD). Each patient received a consensus diagnosis of AD by 2 psychiatrists according to the $10^{\text {th }}$ revision of the International Statistical Classification of Diseases and Related Health Problems (ICD-10). ${ }^{22}$ The most recent alcohol intake was at least 1 week priorly. The exclusion criteria were: age of $<18$ years, a history of a significant psychiatric comorbidity according to the ICD-10. ${ }^{22}$

\section{Data collection}

This study used a structured self-reported questionnaire that was designed to measure the sociodemographic and clinical characteristics of the study participants (gender, age, education, employment status, marital status, place of living, and cigarette smoking). The study participants were assured of the confidentiality of the data obtained. The researcher remained present during the completion of the questionnaires in order to address the participants' questions and to make sure the respondents understood all of the items. Patients who answered "yes" to the following question: "Have you smoked at least 100 cigarettes during your lifetime?" were considered smokers. 
The current severity of drinking was measured using the Alcohol Use Disorders Identification Test (AUDIT) with a Cronbach's alpha index of $0.85 .^{23}$

The ACEs were measured with a tool designed for this study, named the ACE (13) Score. The first 10 questions, developed by Kaiser Permanente and the Centers for Disease Control and Prevention, evaluated exposure to abuse and family dysfunction occurring during the first 18 years of one's life (the ACE Study score). ${ }^{18}$ These 10 questions focused on chronic physical, verbal and sexual abuse, neglect, the loss of one or both parents for any reason (i.e., divorce, separation or death), exposure to domestic violence, and growing up in a household with mental illness, alcohol abuse, drug abuse, or incarceration. The 3 additional questions concerned events that also took place in one's life before the age of 18 and included the following: witnessing a family member's attempted suicide, witnessing a family member's death due to any cause and witnessing a stranger's death due to any cause (e.g., traffic accident). The details of our statistical analysis allow for our results to still be comparable with the studies based on the ACE Study score.

Health behaviors were assessed with the Health Behavior Inventory (HBI) designed by Juczyński. ${ }^{24}$ Internal reliability for the total HBI measured by Cronbach's alpha was estimated at 0.85 and ranged between 0.6 and 0.65 for its 4 subscales. ${ }^{24}$ The HBI comprises 24 statements used to assess health behaviors on 4 subscales: positive attitude (PA), proper dietary habits (PDH), health-related practices (HP), and preventive behaviors $(\mathrm{PB})$. Total HBI scoring falls in the range of 24-120 points, i.e., the respondent must specify on a 5 -point scale how often he/she performed a certain action over the previous year $(1=$ almost never, 2 = rarely, 3 = sometimes, $4=$ often, and $5=$ almost always). The higher the score, the higher the level of health-oriented behaviors. For further interpretation, HBI scoring may be converted into standardized units on a sten scale, which is a sectional scale with an average sten score of 5.5 and a standard deviation of 2 , ranging from 1 to 10 . It was proposed to adopt the following sten ranges: results 1-4 (low score), 5-6 (average score) and 7-10 (high score). ${ }^{24}$

Self-efficacy was measured with the Polish version of the Generalized Self-Efficacy Scale (GSES) by Schwarzer, Jerusalem and Juczyński. A 10-point psychometric scale was used to assess optimistic self-beliefs in coping with a variety of difficult demands in life. ${ }^{24}$ The GSES, with a Cronbach's alpha index of 0.85 for internal reliability, was created to assess a general sense of perceived self-efficacy and to predict the ability to cope with daily struggles and adaptation after experiencing all kinds of stressful life events. ${ }^{24}$ Responses are marked on a 4-point scale for each item and total scoring ranges from 10 to 40 points. The higher the score, the greater the individual's generalized sense of self-efficacy. Scoring $\leq 24$ points is interpreted as a low outcome, between 25 and 29 points as medium outcome, and $\geq 30$ points as high outcome. ${ }^{24}$
In order to address a possible bias connected with participants' intentional attempts to present themselves in either a better or worse mental and general condition, the researcher who remained present during the completion of the questionnaires was not involved in the patients' therapy. Recall bias was still possible during the ACE (13) Score completion, which is listed among the limitations of the study.

\section{Ethics}

All of the participants gave written informed consent for their participation in the study. The study was approved by the Local Bioethics Committee (No. RNN/467/13/KB and $\mathrm{KB} / 843 / 13 / \mathrm{P})$. The study was carried out in accordance with the ethical standards laid down in the 1964 Declaration of Helsinki and its later amendments.

\section{Statistical analysis}

Statistical analysis was performed using STATISTICA v. 12.0 (StatSoft Polska, Kraków, Poland). Generally, a p-value of $<0.05$ was considered significant. The normality of data distribution was evaluated with the ShapiroWilk test. Parameters with normal distribution (age and HBI) are presented as the mean and standard deviation (SD). If the distribution was other than normal, the median and range (min-max) were provided.

The multiple stepwise regression analysis was employed to evaluate the influence of continuous and categorical variables on the GSES and HBI. Variables included in the analysis were selected based on a literature review as possibly associated with self-efficacy and health habits, and were as follows: age, gender, educational level, marital status, occupational status, and place of living. ${ }^{25-27}$ Additionally, for both the GSES and the HBI model, we included the number of ACEs determined by the ACE (13) Score.

In both analyses, the automatic forward selection of variables included in the model was applied. Thus, at the beginning there were no variables in the model, so in the process of testing the algorithm, the variables which improved the model the most were added. This procedure was repeated until no additional variable improved the model.

\section{Results}

\section{Characteristics of patients with alcohol dependence}

The sociodemographic characteristics of 196 patients with AD $(F=50)$ are presented in Table 1 . The mean age of the patients was $43.8 \pm 10.7$ years. Approximately $40 \%$ of the patients with AD were high school graduates (38.8\%) and over half were unemployed (60.2\%). Over 30\% of the patients with AD were divorced (31.2\%). The severity of drinking, measured by the AUDIT interview, was $27.2 \pm 7.6$ out of possible 40 points (Table 1 ). 
Table 1. Socio-demographic and clinical characteristics among patients with AD

\begin{tabular}{|c|c|c|}
\hline \multicolumn{2}{|c|}{ Characteristic } & $\begin{array}{l}\text { Patients with AD } \\
\qquad(n=196)\end{array}$ \\
\hline \multicolumn{2}{|c|}{ Age [years], mean $\pm S D$} & $43.8 \pm 10.7$ \\
\hline \multicolumn{2}{|c|}{ AUDIT score, median (range) } & $28.0(6.0-40.0)$ \\
\hline \multicolumn{2}{|l|}{ Smokers' ${ }^{1}, \mathrm{n}(\%)$} & $180(91.8)$ \\
\hline $\begin{array}{l}\text { Educational } \\
\text { level, n (\%) }\end{array}$ & $\begin{array}{c}\text { basic } \\
\text { vocational } \\
\text { secondary } \\
\text { higher }\end{array}$ & $\begin{array}{l}50(25.5) \\
49(25.0) \\
76(38.8) \\
21(10.7)\end{array}$ \\
\hline $\begin{array}{l}\text { Marital status, } \\
\mathrm{n}(\%)\end{array}$ & $\begin{array}{c}\text { single } \\
\text { married } \\
\text { divorced } \\
\text { widowed }\end{array}$ & $\begin{array}{c}77(39.3) \\
42(21.4) \\
63(32.1) \\
14(7.1)\end{array}$ \\
\hline $\begin{array}{l}\text { Occupational } \\
\text { status, n (\%) }\end{array}$ & $\begin{array}{c}\text { employed } \\
\text { unemployed } \\
\text { retired or survivor sickness }\end{array}$ & $\begin{array}{l}44(22.4) \\
118(60.2) \\
34(17.3)\end{array}$ \\
\hline $\begin{array}{l}\text { Place of living, } \\
\mathrm{n}(\%)\end{array}$ & $\begin{array}{l}\text { village } \\
\text { town } \\
\text { city }^{3}\end{array}$ & $\begin{array}{c}12(6.1) \\
20(10.2) \\
164(83.7)\end{array}$ \\
\hline
\end{tabular}

AD - alcohol dependence; AUDIT - Alcohol Use Disorders Identification Test; ${ }^{1}$ smoker - respondent who smoked at least 100 cigarettes during their lifetime; ${ }^{2}$ town - place of living of $<50,000$ citizens; ${ }^{3}$ city - place of living of $>50,000$ citizens.

\section{ACE (13) Score, $\mathrm{HBI}$ and GSES}

The patients reported from 3 to 10 ACEs (median: 3). Figure 1 presents the reporting frequency of each ACE in both females and males.

On the HBI scale, the patients' mean score was $64.4 \pm 16.6$ out of 120 points. Within this scale, scores on the subscales for PDH ranged from 6 to 30 points (median: 14); for PB they also ranged from 6 to 30 points (median: 17); for PA they varied between 6 and 29 points (median: 18); and for HP, between 6 and 28 points (median: 15.5).

On the GSES scale, patients scored from 10 to 40 points (median: 30 ) out of 40 possible points.

\section{Multiple stepwise regression analysis for variation in health habits assessed by the $\mathrm{HBI}$ and self-efficacy assessed by the GSES}

The model for the GSES explained $6.8 \%$ of its variability (Table 2$)$. Gender $(\beta=-2.176 ; p=0.036)$ and the number of ACEs $(\beta=-0.377 ; p=0.026)$ were significant determinants. Although marital status, educational level and place of living were not significant determinants in the stepwise method, they entered the final model. The ACEs explained $3.2 \%$ of the variability of general self-efficacy assessed by the GSES.

The model for the HBI explained $11.2 \%$ of its variability (Table 2). Age $(\beta=0.336 ; p=0.008)$, place of living $(\beta=7.481$; $\mathrm{p}<0.001)$ and the number of ACEs $(\beta=-1.210 ; \mathrm{p}=0.007)$ were significant determinants; however, marital status also entered the model. Here, the ACEs explained 3.9\% of the variability of health behaviors assessed by the HBI.
Table 2. The multiple stepwise regression analysis for variation in health habits assessed by the HBI and general self-efficacy assessed by the GSES in patients with $A D$

\begin{tabular}{|l|c|c|c|c|}
\multirow{2}{*}{\multicolumn{1}{|c|}{ Variable }} & \multicolumn{2}{c|}{ GSES } & \multicolumn{2}{c|}{ HBI } \\
\cline { 2 - 5 } & $\begin{array}{c}\beta \text { coef- } \\
\text { ficient }\end{array}$ & $p$-value & $\begin{array}{c}\beta \text { coef- } \\
\text { ficient }\end{array}$ & $p$-value \\
\hline Gender & -2.178 & $0.036^{*}$ & -0.406 & 0.883 \\
\hline Age & -0.025 & 0.703 & 0.368 & $0.004^{*}$ \\
\hline Occupational status & 0.117 & 0.879 & 1.809 & 0.371 \\
\hline Marital status & -0.676 & 0.128 & -1.817 & 0.176 \\
\hline Educational level & 0.651 & 0.150 & 1.253 & 0.294 \\
\hline Place of living & 1.065 & 0.195 & 7.481 & $<0.001^{*}$ \\
\hline ACE (13) Score & -0.377 & $0.026^{*}$ & -1.21 & $0.007^{*}$ \\
\hline
\end{tabular}

AD - alcohol dependence; GSES - General Self-Efficacy Scale; HBI - Health Behavior Inventory; ${ }^{*}$ statistical significance.

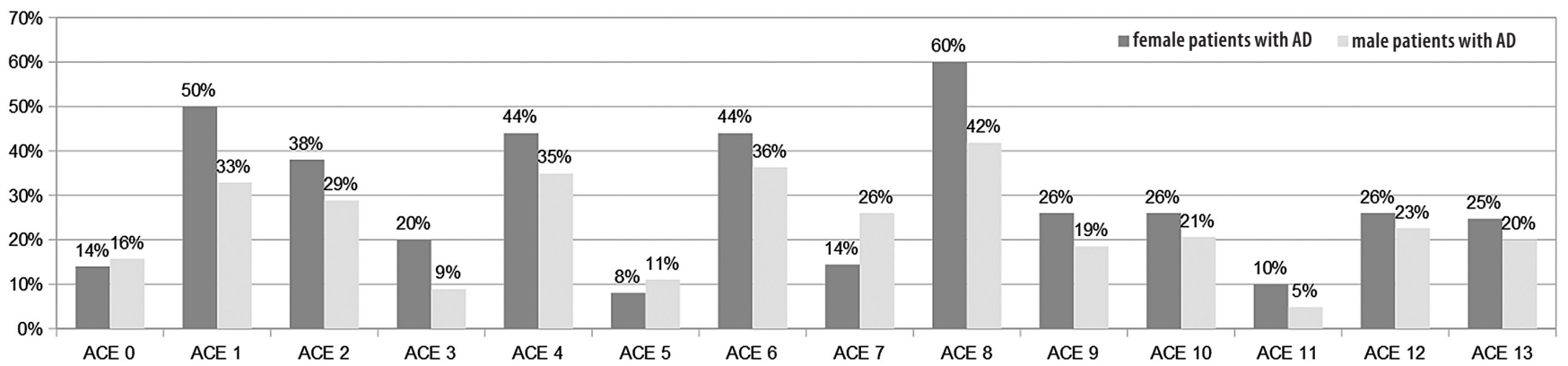

Fig. 1. Prevalence of ACEs in patients with $A D(n=196)$

The categories with an ACE (13) Score are as follows: ACE1 - psychological abuse; ACE2 - physical abuse; ACE3 - sexual abuse; ACE4 - emotional neglect; ACE5 - physical neglect; ACE6 - loss of contact with parents; ACE7 - witnessing physical abuse toward one's mother or stepmother; ACE8 - drinking/ alcohol/drug use by a household member; ACE9 - mental illness/suicide attempt of a household member; ACE10 - incarceration of a household member; ACE11 - witnessing a family member's suicide attempt; ACE12 - witnessing a family member's death; ACE13 - witnessing a stranger's death. 


\section{Discussion}

This study examined health behaviors, general self-efficacy and self-reported childhood adversities in patients with AD. We found the ACEs to be among the factors that significantly and adversely influenced general selfefficacy and promoted health-harming behaviors in adulthood in patients with AD.

The explanatory power of the model of health behaviors for patients with AD as used in this study was found to be $11.2 \%$. The ACEs, current age and place of living were variables with a significant association with health habits (Table 2). Out of all of these variables, the ACEs explained $3.9 \%$ of the HBI variability.

The literature on health harm associated with alcohol drinking states that there is no obvious positive association between alcohol intake and health harm, as health harm is already a final result associated at least partially with alcohol misuse and other health-harming behaviors, such as cigarette smoking, poor diet and poor voluntary exercise. ${ }^{17,28}$ Cross-sectional studies relying on self-reporting may provide underestimated data on the duration and severity of drinking, or show less severe alcohol drinking in patients with existing alcohol-related health harm, i.e., head and neck cancer or esophageal cancer, without a lifetime drinking history and previous drinking patterns. ${ }^{29,30}$ Health-harming behaviors also decreased with age of our patients, which may suggest that they improved their health behaviors because of alarming health costs, or as a result of support from family or healthcare providers. Our study did not aim to assess current health harm that was potentially associated with alcohol misuse, but current health habits in patients with AD. Our results are in line with other studies that show a parallel coexistence of alcohol misuse and other health-harming behaviors. Approximately 92\% of our patients with AD were also cigarette smokers. Bellis et al. confirmed strong associations between alcohol misuse or smoking and low levels of exercise or poor diet. ${ }^{16}$ A meta-analysis by Probst et al. confirmed the accumulation of health-harming behaviors in individuals with alcohol misuse. ${ }^{31}$ However, they stated that this accumulation was predominantly reserved for communities living in socioeconomic deprivation and they considered healthcare provision, dietary habits and smoking behaviors as putative main factors underlying this phenomenon. ${ }^{31}$ According to their analysis, low socioeconomic status was related to malnutrition, i.e., to purchasing foods that are high in fat, salt and sugar, processed food and fast food consumption, and low fruit and vegetable consumption, which may interact with alcohol consumption to alter protein and vitamin absorption and increase the risk of vitamin and mineral deficiencies. ${ }^{31}$ In our study, occupational status and educational level did not enter the model of variability in health behaviors, as there were probably factors other than education, i.e., factors related to the place of living, which implicated health habits. There might have been better availability of healthcare and health education, and better promotion of voluntary exercise and a healthy diet in cities than in towns and villages. Health habits were more health-oriented in the city-dwelling patients, less health-oriented among the inhabitants of towns, and the least among village inhabitants. However, the ACEs were found to correlate with them adversely, i.e., the higher the number of ACEs, the more health-harming habits were present in the patients.

The ACEs were also considered a measure of social inequities and were more frequent in communities of poor socioeconomic status. ${ }^{32}$ A USA national study that applied the ACE Study score in a sample of the general population found that the rate of premature death ( $<65$ years of age) rose along with the number of self-reported ACEs. ${ }^{18,19} \mathrm{Re}-$ spondents who had experienced at least 4 ACEs of any type were about twice as likely to have an elevated prevalence of premature death relative to the respondents with no such experience. The highest risk occurred among those who reported having been physically neglected and living with substance-abusing or criminal family members during their childhood. ${ }^{17}$

The explanatory power of the model of general self-efficacy for our patients was found to be $6.8 \%$ (Table 2). Only the ACEs and gender of the study participants were found to be significantly associated with general self-efficacy; the ACEs explained 3.2\% of GSES variability.

In our model of general self-efficacy variability, being a female patient and reporting a higher number of ACEs was significantly associated with lower general self-efficacy. As pointed out by Bandura et al., general self-efficacy may be impaired by previously experienced life adversities. ${ }^{8}$ The kind of ACE category and individual susceptibility to ACEs may also be meaningful for future general selfefficacy. The female patients with AD were more likely to report both psychological and sexual abuse, and a household member's alcohol or drug misuse. General self-efficacy was referred to as global confidence in one's coping with demanding or novel situations. It warrants dealing with a variety of stressful situations and characterizes one's social skills. ${ }^{4}$ Lower general self-efficacy was found to be related with a higher risk of suicide ideation. ${ }^{33,34}$ The majority of studies on self-efficacy in substance abuse disorders have focused on skill-oriented self-efficacy as an important factor which influences treatment outcome. Skill-oriented selfefficacy, i.e., the ability to not drink in high-risk situations, is associated with an effective use of coping strategies and with lower relapse rates. ${ }^{6}$ As reviewed by Trucco et al., there has been a growing number of studies which indicate that individuals with higher skill-oriented self-efficacy scores are less likely to relapse and more likely to remain abstinent up to 6 months following treatment, and, similarly, that low skill-oriented self-efficacy was predictive of a relapse during 12 months following inpatient alcohol detoxification. ${ }^{7}$ As assessed by Czyz et al., lower self-efficacy was reported by subjects with substance abuse disorders who had more severe suicidal ideation and by those with more 
suicide attempts. ${ }^{33}$ The explanatory power of our model of general self-efficacy for our patients was found to be only $6.8 \%$. Alcohol consumption is a known factor which promotes a phenotype of loss of behavioral flexibility; thus, alcohol misuse may be primary to social impairment and may promote social anxiety, altered adult synapses, altered cognition, reduced adult neurogenesis, and increased neuroimmune gene expression and the epigenetic modifiers of gene expression. ${ }^{1}$ Since the adolescent brain is uniquely sensitive to alcohol neurotoxicity, decreased adult neurogenesis might contribute to an increased risk of adult psychopathology and cognitive dysfunction. ${ }^{1}$ As reviewed by Crews et al., ethanol exposure in early adolescence adversely affected social skills of rats, particularly males, in terms of social inhibition, although it occurred in a lesser degree in adolescents than in adult rats. ${ }^{1} \mathrm{~A}$ meta-analysis by Bora and Zorlu showed that patients with alcohol abuse disorders presented significantly impaired facial emotion recognition, particularly for disgust and anger. ${ }^{2}$ Further studies on general self-efficacy and its relationship to ACEs should utilize more psychometric analyses and functional neuroimaging.

The World Health Organization pointed to ACEs as an important target for primary preventive strategies of chronic ill health during one's lifetime. ${ }^{35}$ Our study found ACEs to be one of the factors that significantly correlate with general self-efficacy and health habits in patients with $\mathrm{AD}$ and indicate that they should be targeted with primary preventive strategies for better overall health in adulthood. However, in case it is already too late for primary preventive strategies, secondary prevention to improve general self-efficacy impaired by childhood adversity should be introduced. The cognitive-behavioral theory posits that higher confidence in the ability to not drink in a high-risk situation is associated with the effective use of coping strategies and lower relapse rates. ${ }^{6}$

\section{Limitations}

The main limitation of studies on childhood adversities, including ours, is that the analyzed data is retrospective and self-reported, and that recall bias is still possible; for example, the respondent may recall more negative autobiographical adversity when he or she is answering the questions regarding poor mental and physical health conditions in adulthood. ${ }^{36}$ Thus, it is possible that variables identified in later life as factors possibly influencing general well-being in adulthood would not prove to be predictive of ultimate health outcomes when assessed in earlier stages of life. ${ }^{20}$ Moreover, the paucity of factors that might have been either supportive or harming to the respondent and were not included in the study (i.e., a parent's hidden emotional problems or receiving professional help) could influence the respondent's current health. The cross-sectional design of the study precludes both causal inference (as event-reporting may be confounded by the current psychological condition and age) and a longitudinal analysis of adjustment methods ${ }^{37}$ Finkelhor et al. also considered another important limitation of studies analyzing retrospectively-recalled ACEs. ${ }^{20}$ Between older and younger respondents, there may be a difference in pointing to childhood experiences as adversities or not, which mirrors social changes regarding the norms and awareness of childhood experiences. Thus, in the younger cohort of respondents, due to the increased availability of professional support (i.e., a psychotherapist at school) and more cultural awareness, parental divorce may be nonpredictive of a worse health outcome in adulthood than may be the case in an older cohort. ${ }^{20}$

\section{Conclusions}

We confirmed that childhood adversities, understood as neglect, trauma, physical and psychological abuse, and witnessing someone's death, are one of the factors significantly associated with the development of health-harming behaviors and worse general self-efficacy in adult patients with AD. Even though these were among many other factors influencing health habits and general self-efficacy, and explained only approx. $4 \%$ of the variability of health habits and about $3.5 \%$ of the variability of general self-efficacy, the study suggests the need for psychotherapy to focus on childhood trauma while strengthening general selfefficacy, targeted to improve alcohol abstinence.

\section{References}

1. Crews FT, Vetreno RP, Broadwater MA, et al. Adolescent alcohol exposure persistently impacts adult neurobiology and behavior. Pharmacol Rev. 2016;68(4):1074-1109.

2. Bora E, Zorlu N. Social cognition in alcohol use disorder: A metaanalysis. Addiction. 2017;112(1):40-48. doi:10.1111/add.13486

3. Thorberg FA, Young RM, Lyvers M, et al. Alexithymia in relation to alcohol expectancies in alcohol-dependent outpatients. Psychiatry Res. 2016;236:186-188. doi:10.1016/j.psychres.2016.01.016

4. Schwarzer R, Mueller I, Greenglass E. Assessment of perceived general self-efficacy on the internet: Data collection in cyberspace. Anxiety Stress Coping. 1999;12:145-161.

5. Bandura A. Social Foundations of Thought and Action: A Social Cognitive Theory. Englewood Cliffs, NY: Prentice-Hall; 1986.

6. Greenfield SF, Hufford MR, Vagge LM, et al. The relationship of selfefficacy expectancies to relapse among alcohol dependent men and women: A prospective study. J Stud Alcohol. 2000;61(2):345-351.

7. Trucco EM, Connery HS, Griffin ML, et al. The relationship of selfesteem and self-efficacy to treatment outcomes of alcohol-dependent men and women. Am J Addict. 2007;16(2):85-92.

8. Bandura A, Reese $L$, Adams NE. Microanalysis of action and fear arousal as a function of differential levels of perceived self-efficacy. J Pers Soc Psychol. 1982;43:5-21.

9. Benight C, Bandura A. Social cognitive theory of posttraumatic recovery: The role of perceived self-efficacy. Behav Res Ther. 2004;42(10): 1129-1148. doi:10.1016/j.brat.2003.08.008

10. Hinnen C, Sanderman R, Sprangers MAG. Adult attachment as mediator between recollections of childhood and satisfaction with life. Clin Psychol Psychother. 2009;16:10-21. doi:10.1002/cpp.600

11. Suzuki H, Tomoda A. Roles of attachment and self-esteem: Impact of early life stress on depressive symptoms among Japanese institutionalized children. BMC Psychiatry. 2015;15:8. doi:10.1186/s12888015-0385-1 
12. Felitti VJ. The relationship of adverse childhood experiences to adult health: Turning gold into lead [in German]. Z Psychosom Med Psychother. 2002;48(4):359-369.

13. Anda RF, Whitfield CL, Felitti VJ, et al. Adverse childhood experiences, alcoholic parents, and later risk of alcoholism and depression. Psychiatr Serv. 2002;53:1001-1009.

14. Shin SH, Pelucchi C, Bagnardi V, et al. Child abuse and neglect: Relations to adolescent binge drinking in the national longitudinal study of adolescent health (AddHealth) study. Addict Behav. 2009;34: 277-280.

15. Latendresse SJ, Rose RJ, Viken RJ, et al. Parenting mechanisms in links between parents' and adolescents' alcohol use behaviors. Alcoholism Clin Exp Res. 2008;32:322-330. doi:10.1111/j.1530-0277.2007.00583.x

16. Bellis MA, Hughes $K$, Leckenby $N$, et al. Measuring mortality and the burden of adult disease associated with adverse childhood experiences in England: A national survey. J Public Health (Oxf). 2015;37(3): 445-454.

17. Bellis MA, Hughes $K$, Nicholls J, et al. The alcohol harm paradox: Using a national survey to explore how alcohol may disproportionately impact health in deprived individuals. BMC Public Health. 2016;16:111. doi:10.1186/s12889-016-2766-x

18. Felitti VJ, Anda RF, Nordenberg D, et al. Relationship of childhood abuse and household dysfunction to many of the leading causes of death in adults: The Adverse Childhood Experiences (ACE) Study. Am J Prev Med. 1998;14:245-258.

19. Anda RF, Dong M, Brown DW, et al. The relationship of adverse childhood experiences to a history of premature death of family members. BMC Public Health. 2009;9:106. doi:10.1186/1471-2458-9-106

20. Finkelhor $D$, Shattuck $A$, Turner $H$, et al. Improving the adverse childhood experiences study scale. JAMA Pediatr. 2013;167(1):70-75. doi:10. 1001/jamapediatrics.2013.420

21. Stathopoulou G, Powers M, Berry A, et al. Exercise interventions for mental health: A quantitative and qualitative review. Clin Psychol Sci Pract. 2006;13:180-193.

22. World Health Organization. The ICD-10 Classification of Mental and Behavioral Disorders: Clinical Description and Diagnostic Guidelines. Geneva, Switzerland: WHO; 1992.

23. Daeppen JB, Yersin B, Landry U, et al. Reliability and validity of the Alcohol Use Disorders Identification Test (AUDIT) imbedded within a general health risk screening questionnaire: Results of a survey in 332 primary care patients. Alcohol Clin Exp Res. 2000;24(5):659-665.

24. Juczyński Z. Instruments for Measurement in Health Promotion and Health Psychology [in Polish]. $2^{\text {nd }}$ ed. Warsaw, Poland: Pracownia Testów Psychologicznych; 2012.
25. Schieman S, Taylor J. Statuses, roles, and the sense of mattering. Sociol Perspect. 2001;44(4):469-484. doi:10.1525/sop.2001.44.4.469

26. McCormack L, Haun J, Sørensen K, Valerio M. Recommendations for advancing health literacy measurement. J Health Commun. 2013;18(1): 9-14.

27. Weaver NL, Wray RJ, Zellin S, et al. Advancing organizational health literacy in health care organizations serving high-needs populations: A case study. J Health Commun. 2012;17(3):55-66.

28. Dawson DA, Li TK, Grant BF. A prospective study of risk drinking: At risk for what? Drug Alcohol Depend. 2008;95:62-72.

29. Roerecke $M$, Rehm J. Irregular heavy drinking occasions and risk of ischemic heart disease: A systematic review and meta-analysis. Am J Epidemiol. 2010;171:633-644.

30. Rehm J, Patra J, Popova S. Alcohol drinking cessation and its effect on esophageal and head and neck cancers: A pooled analysis. Int J Cancer. 2007;121:1132-1137.

31. Probst $C$, Roerecke M, Behrendt S, et al. Socioeconomic differences in alcohol-attributable mortality compared with all-cause mortality: A systematic review and meta-analysis. Int J Epidemiol. 2014;43(4): 1314-1327. doi:10.1093/ije/dyu043

32. Marmot M, Allen J, Bell R, et al.; Consortium for the European Review of Social Determinants of Health and the Health Divide. WHO European review of social determinants of health and the health divide. Lancet. 2012;380(9846):1011-1029.

33. Czyz EK, Bohnert ASB, King CA, et al. Self-efficacy to avoid suicidal action: Factor structure and concurrent validity among adults in substance use disorder treatment. Suicide Life Threat Behav. 2014;44(6): 698-709. doi:10.1111/sltb.12101

34. Kobayashi Y, Fujita K, KanekoY, et al. Self-efficacy as a suicidal ideation predictor: A population cohort study in rural Japan. Open Journal Prev Med. 2015;5:61-71.

35. World Health Organization. Global Action Plan for the Prevention and Control of Noncommunicable Diseases 2013-2020. http:/www. who.int/nmh/publications/ncd_action_plan/en/. Published 2013. Accessed December 16, 2015.

36. Hardt J, Rutter M. Validity of adult retrospective reports of adverse childhood experiences: Review of the evidence. J Child Psychol Psychiatry. 2004;45(2):260-273.

37. Layne CM, Warren JS, Hilton S, et al. Measuring Adolescent Perceived Support Amidst War and Disaster: The Multi-Sector Social Support Inventory. In: Baker BK, ed. Adolescents and War: How Youth Deal With Political Violence. New York, NY: Oxford University Press; 2009:145-176. 